\title{
Associations between cognitive abilities and life satisfaction in the oldest-old. Results from the longitudinal population study Good Aging in Skåne
}

This article was published in the following Dove Press journal:

Clinical Interventions in Aging

10 July 2013

Number of times this article has been viewed

\section{Åsa Enkvist \\ Henrik Ekström \\ Sölve Elmståhl}

Department of Health Sciences, Division of Geriatric Medicine, Lund University, Skåne University Hospital, Malmö, Sweden
Correspondence: Åsa Enkvist Department of Health Sciences, Division of Geriatric Medicine, Lund University, Skåne University Hospital, SE-205 02

Malmö, Sweden

Tel +46 40 39l 000

Fax +46 40 391 313

Email asa.enkvist@med.lu.se
Introduction: Studies on the associations between cognitive abilities and life satisfaction (LS) in the oldest-old are few. The aim of this study was to explore whether abilities in six different cognitive domains could predict LS in the oldest-old 3 years later.

Methods: The study population consisted of 681 individuals aged 78-98 years, drawn from the longitudinal population study "Good Aging in Skåne," which is part of a national survey (The Swedish National Study on Aging and Care). Scores on 13 cognitive tests were related to scores on Neugartens' LS index A (LSI-A) 3 years later. The cognitive tests were added into six different cognitive domains. A multiple regression analysis was constructed for each cognitive domain separately, with scores on the LSI-A as the dependent variable. The model was adjusted stepwise for sex, age, education, functional capacity, and depressive mood.

Results: Significant correlations were found between digit cancellation, word recall, verbal fluency (VF) A, VF animals, VF occupations, and mental rotations at baseline, as well as LSI-A at follow-up. The domains of spatial abilities $(\mathrm{B}=0.453, P=0.014)$ and processing speed $(\mathrm{B}=0.118, P=0.020)$ remained significantly associated with LSI-A 3 years later after adjustment.

Conclusion: The cognitive domains of spatial abilities and processing speed predicted LS 3 years later in the oldest-old. Clinical implications are discussed.

Keywords: oldest-old, life satisfaction, longitudinal, crystallized and fluid intelligence, cognition

\section{Introduction}

As the part of the population called the oldest-old is growing in number, geriatric research has partly changed focus from being preoccupied with disease and disability to focusing on life satisfaction (LS), psychological well-being, successful aging, and other related concepts. Multiple areas have been shown to influence LS in this age group - health status, burden of symptoms, functional ability, personality factors, and marital status are some examples. ${ }^{1-3}$ The oldest-old is the group in society with the highest risk of functional decline - physical as well as cognitive. Therefore, it is of importance to explore the associations between not only physical, but also cognitive functioning and positive health outcomes such as LS, quality of life, and other related concepts. Few studies have explored the associations between LS and cognition longitudinally in the oldest-old. ${ }^{4}$

Jones et $\mathrm{al}^{5}$ found higher cognitive functioning to be positively related to LS and positive affectivity. The authors highlighted the clinical relevancy in establishing the link between subjective well-being and objectively measured cognitive performance. They speculated 
that people with higher cognitive abilities would be better able to appreciate the subtle yet positive aspects of living, or that people are better fit to utilize resources and adapt to circumstances, thus facilitating happiness. The authors also pointed to the idea that many gerontological studies on LS and subjective well-being exclude persons with any degree of cognitive impairment. This makes the results easier to interpret, but also limits the study population to extremely healthy individuals, and is not totally representative of elderly people.

St John and Montgomery ${ }^{6}$ found that people with cognitive impairment had lower LS than people scoring in the normal range of the extended version of the Mini-Mental State Examination (MMSE) that was used. The association between LS and cognitive ability was attenuated when adjusted for functional capacity and depressive symptoms, and the latter two factors seemed to influence LS stronger than cognitive abilities. The authors commented on the fact that studies of the effects of cognitive abilities on LS in the general older population are few and that most studies that explore associations between LS and cognition, concern only people with dementia and/or use methods such as the MMSE that only detect a rather marked cognitive loss.

Through researchers such as Baltes, ${ }^{7}$ views on the aging mind have shifted from a preoccupation with cognitive decline to a perspective including potentials for growth. In his studies, he categorizes the mind into two domains - crystallized and fluid intelligence (Gc and Gf, respectively), ${ }^{8}$ which have been illustrative in that they display different lifespan trajectories. Gf shows a constant decline after peaking in young adulthood, while Gc remains stable or may even increase throughout the lifespan. In light of this, the findings of Wolinsky et al, ${ }^{9}$ who conducted cognitive training in domains related to Gf, are interesting. Healthy individuals above the age of 65 years were trained in three different cognitive domains: processing speed, memory, and reasoning. The results showed that individuals who had been trained in processing speed were $30 \%$ less likely to experience clinically relevant increases in depressive symptoms than either the control group or those trained in the other domains, and this effect remained after 5 years. Siedlecki et $\mathrm{al}^{10}$ also studied the influence of Gc and Gf on adults of different ages from this perspective. The authors hypothesized that Gc would be increasingly important for LS with increasing age, while the significance of Gf would diminish with age as the individual partakes less in workplace situations and career management. In the results, however, Gc was not associated to LS at any age, while Gf was associated with the younger group (aged 18-59 years), but not with the older group (aged 60-94 years).
Baltes and Baltes ${ }^{11}$ also defined a psychological model for the study of successful aging - the theory of selective optimization with compensation. "Selection" refers to the individuals' choice to focus on areas of high priority as they face increasing restrictions in functioning due to age related losses. "Optimization" refers to the person investing in behaviors that augment and maximize reserve capacities and the quality of their lives. Finally, as capacities decline, different forms of compensation - psychological as well as technological - become vital. Baltes ${ }^{7}$ showed that cognitive abilities related to fluid as well as Gc can be improved by training, even in advanced ages. Society is facing a huge challenge as the oldest-old segment of the population expands. Baltes ${ }^{7}$ discussed the problems faced as society and individuals strive to optimize living conditions and compensate for the various types of losses faced by the oldest-old, since impaired physical and cognitive abilities might limit the ability to benefit from compensations (eg technological) offered.

Previous studies have either included selective healthy study samples or they have not taken potential confounders into account. The null hypothesis for this study was that cognitive abilities in the oldest-old cannot predict LS 3 years later. The aim of this study was to explore whether results on 13 cognitive tests, administered separately and organized into six cognitive domains, could predict LS 3 years later in the oldest-old. Factors such as stroke, dementia, functional capacity, and depressive mood, known to influence or be influenced by cognitive performance as well as LS, were included as confounders in the model.

\section{Methods \\ Study population}

The study was designed as a longitudinal population-based cohort study with a 3 year follow-up period. ${ }^{12}$ A randomized selection was made from the Swedish population register of the respective age group and a letter of invitation was sent out. Inclusion criteria were that the individuals had to be residents in one of the five municipalities of Skåne, and they had to be between 78 years and 93 years of age. The only exclusion criterion was if the respondent could not understand Swedish. At baseline, the target population was 1,300 individuals; of these, 1,253 individuals - 78 years to 93 years old - participated. Of those, 314 died, eight moved from the area, and 250 chose not to take part in the 3 -year reexamination. The participation rate at baseline was $53.5 \%$, which is similar to the rate of other studies that include the oldest-old. ${ }^{13}$ 
The study population thus consisted of 681 individuals and included six age cohorts at baseline: 78-year-olds, 81-yearolds, 84-year-olds, 87-year-olds, 90-year-olds, and 93-yearolds. Five municipalities from the southern part of Sweden were included: Malmo, Eslöv, Hässleholm, Osby, and Ystad. Participants filled in questionnaires and underwent medical examinations, neuropsychological testing, and functional tests with identical study protocols at both examinations. Examination took place either at the research clinic or in the participants' homes, and the participant received help to fill in the questionnaires by a research assistant if the participant asked for it. Informed consent was obtained from the participant, and when needed (if the participant was cognitively or otherwise impaired), from relatives.

The study was approved by the Ethical Committee at Lund University (LU 744-00).

\section{Questionnaires}

LS was assessed using Neugartens' Quality of Life Scale (LSI-A).${ }^{14}$ It is a multidimensional scale and consists of five components of LS: zest (versus apathy); resolution and fortitude; congruence between desired and achieved goals; positive self-concept; and mood tone. It includes 20 questions, which are scored from 0 to 2 , to which participants had three possible answers to choose from: "disagree," "doubtful," and "agree." A high score thus indicates high LS. The participant is supposed to have a high LS to the extent that he/she: (1) takes pleasure in the activities that constitute his or her everyday life; (2) regards his or her life as meaningful and readily accepts the life that has been; (3) feels he or she has been successful in achieving major goals in life; (4) has a positive self-image; and (5) maintains optimistic attitudes and moods.

The LSI-A was constructed to be a valid and reliable instrument measuring general mental well-being in an elderly population. Cronbach's alpha for internal consistency was established by Neugarten ${ }^{14}$ and later confirmed by Lobello et al, ${ }^{15}$ who found a Cronbach's alpha ranging from $0.85-0.92$.

The cognitive tests were organized into six domains accordingly: $:^{16}$

1. "Executive" (including Trail Making Test B [TMT B], the verbal fluency tests, and confidence).

2. "Processing speed" (including digit cancellation and comparing figures).

3. "Episodic memory" (including word recall).

4. "Spatial" (including mental rotations).

5. "Working memory" (including digit span forwards and backwards).
6. "Semantic memory" (including synonym reasoning block 1 [SRB1]).

TMT B tests executive abilities. In the Good Aging in Skåne project, a shortened version of the original TMT B is used. The participant is asked to draw a line between the correct sequence of numbers and letters in circles on a paper as fast as possible. ${ }^{16}$ The time in seconds required to fulfill the test was measured as the outcome.

Four tests measuring verbal fluency were administered. ${ }^{16}$ In category fluency, participants were asked to generate as many animals and occupations as possible in 60 seconds per category. For the letter fluency tasks, participants were given 60 seconds to generate as many words as they could, beginning with the letters $\mathrm{A}$ and $\mathrm{F}$, respectively.

"Confidence" is a test of the executive abilities which encompasses the ability to plan and organize actions, ${ }^{16}$ and also to evaluate and interrupt actions that do not yield desired results. In this test, ten questions are presented to the participant. Each question contains two sentences, of which only one is correct. Each participant is asked to identify the correct answer and then state how sure he or she is that he or she answered correctly. The range of scores for this test is 1-10, with a higher score indicating more confidence.

"Digit cancellation" measures processing speed; sustained attention, visual scanning, activation and inhibition of responses. ${ }^{16}$ Participants are required to cross out a certain digit (in our case, digit four) among a number of randomly interspersed numbers in rows throughout the course of 30 seconds. The range of scores is $0-43$.

"Comparing figures" is a test that measures perceptual speed and visuospatial ability. ${ }^{16}$ During the course of 30 seconds, the participants are asked to decide whether two figures in different pairs are identical or not; the range of scores is $0-30$.

The "word recall and recognition of positions" test, 16 cards with words on them were presented to the participant one at a time. Each card was presented for 5 seconds. The positions of the words were randomly varied. After the presentation, the participant was asked to recall as many of the words as possible during 2 minutes. The test reflects episodic and working memory. ${ }^{16}$ Later, the same words were presented with an equal number of new words, and the participant was asked to identify those words that were in the original version and to indicate in what position the original word was. The range of scores was 0-16.

"Mental rotations" measures three-dimensional thinking and spatial ability. ${ }^{21}$ For this test, each participant is shown an index figure and three figures, of which one is identical to the index but is rotated; the participant has to identify the latter. The range of scores is $0-10$, with 10 indicating better spatial ability. 
The next test that was used was the digit span test of the Wechsler Intelligence and Memory Scales, used to measure working memory. ${ }^{20}$ The participant is supposed to repeat numbers after the test leader - initially two, then increasing numbers in a row. In the digit span backwards task, numbers are presented and the participant is to repeat as many of the numbers as he or she can in reverse order. The maximum score for both tests is 14 .

Semantic memory was measured with the synonym test SRB $1 .{ }^{17}$ The participant is shown a word to which he or she is supposed to find the synonym among five alternatives. In all, 30 words are presented; the maximum score is 30 , and time limit is set to 7 minutes. The test is not perceived to be age-sensitive and reflects Gc. ${ }^{16}$

Depressive mood was assessed with the Montgomery Åsberg Depression Rating Scale, which is a subscale of the Comprehensive Psychiatric Rating Scale. ${ }^{22}$ This scale includes ten questions encompassing depression, anxiety, sleep, appetite, concentration, initiative, emotional involvement, content of thoughts, and basic mood. Each question is graded in six steps. The score ranges from $0-60$, where 0-6 indicates mental well-being, 7-34 represents mild to moderate depressive mood, and 35-60 indicates severe depressive mood. ${ }^{23}$ In our population, no subject scored above 35 , so this variable could be split into two groups: " 1 " for nondepressive mood $(\mathrm{n}=507)$ and " 2 " for mildly to moderately depressive mood $(n=117)$.

Functional capacity was measured according to the revised version of Sonn and Asberg's activities of daily life (ADL) scale. ${ }^{24}$ The ADL staircase was dichotomized into two groups where subjects reporting total independence were categorized as " 1 " $(n=299)$ and subjects reporting any kind of dependence (eg, for grocery shopping, cleaning, or cooking) were categorized as " 0 " $(n=351)$.

The occurrence of stroke (diagnosed according to the International Classification of Diseases version 10) and dementia (diagnosed according to criteria from the Diagnostic Statistical Manual of Mental Disorders, fourth revision) was retrieved from the medical examination, and medical history and information was obtained from proxy and medical records. Subjects were categorized as "yes" or "no" for having had each of the conditions. Thirty-eight subjects had a diagnosis of dementia and 73 had a diagnosis of stroke.

The participants' level of education was categorized into two groups for stratification and three groups for the regression model. Groups were defined depending on whether the participant had fulfilled elementary school $(\mathrm{n}=436)$, high school $(n=158)$, and/or university $(n=73)$.
Considering any misclassification due to test performance, a sensitivity test was carried out regarding the correlations between LS and the 13 cognitive tests. During the cognitive tests, the test leader filled in a protocol registering whether problems with eyesight $(n=92)$, hearing $(n=85)$, motor skills $(n=51)$, or communication difficulties $(n=28)$ interfered with the testing. The correlations were made again after excluding this group, and results did not differ from the total study population. Therefore, in the subsequent analyses, subjects with eyesight, hearing, motor, and communication problems were included.

\section{Statistical methods}

To make scales comparable when organized into domains, the scales were transformed using $z$-standardization according to the following formula:

$$
z=\mathrm{x}_{\mathrm{i}}-\mathrm{m} / \mathrm{SD}
$$

where $\mathrm{x}_{\mathrm{i}}$ is the individual, $\mathrm{m}$ is the mean, and SD is the standard deviation.

Differences in the means between the group that came to the reexamination and the drop-out group were tested with Student's $t$-test (Table 1). The LSI-A and the cognitive test results were normally distributed. Correlations between cognitive variables at baseline and LSI-A at follow-up were tested using Pearson's correlation coefficient (Tables 2 and 3 ). Correlations were calculated for the whole sample, and were stratified for known confounders including stroke, depressive mood, low/high education, and functional ability.

Separate multiple linear regression models with cognitive domains at baseline and LSI-A at reexamination as the dependent variable were constructed (Table 4). Each model was adjusted for known confounders, with each predictor variable entered into each successive model accordingly, including sex, age, education, functional capacity, and depressive mood. Regression coefficients and $P$-values were calculated. Data analysis was performed using SPSS software version 20 (IBM Corporation, Armonk, NY, USA).

\section{Results}

The study population consisted of 418 women and 263 men; 488 individuals were between 78-84 years old, while 193 were between $87-93$ years old. A total of 584 participants represented urban living, and 87 represented rural living (information was missing for ten individuals). In addition, 436 individuals had an education level equal to or less than 9 years, while 231 had studied for more than 9 years (data from 14 participants were missing). 
Table I Results on cognitive tests, mean and SD at baseline for the study population and dropouts

\begin{tabular}{|c|c|c|c|c|c|c|}
\hline \multirow[t]{2}{*}{ Cognitive variable } & \multicolumn{2}{|c|}{ Study population } & \multicolumn{2}{|l|}{ Dropout } & \multirow[t]{2}{*}{$P$-value } & \multirow[t]{2}{*}{ Range } \\
\hline & Mean (SD) & $\mathbf{n}$ & Mean (SD) & $\mathbf{n}$ & & \\
\hline Age (years) & $83.0(4.4)$ & 681 & $85.4(4.8)$ & 572 & $<0.001$ & - \\
\hline Trail Making Test B (A) & $52.8(40.5)$ & 560 & $61.6(4 I .9)$ & 345 & $<0.002$ & - \\
\hline Verbal fluency $F(A)$ & $12.6(5.2)$ & 624 & $10.9(5.1)$ & 428 & $<0.001$ & - \\
\hline Verbal fluency $A(A)$ & $9.8(4.9)$ & 623 & $8.2(4.5)$ & 425 & $<0.001$ & - \\
\hline Verbal fluency, animals (A) & $17.3(5.8)$ & 628 & $14.9(5.8)$ & 434 & $<0.001$ & - \\
\hline Verbal fluency, occupations (A) & $12.8(4.6)$ & 621 & $10.6(4.4)$ & 429 & $<0.001$ & - \\
\hline Confidence (A) & $6.4(1.6)$ & 627 & $6.1(1.6)$ & 432 & $<0.001$ & $0-10$ \\
\hline Comparing figures (B) & $9.8(3.2)$ & 569 & $8.6(3.3)$ & 355 & $<0.001$ & $0-30$ \\
\hline Digit cancellation (B) & I4.3 (3.8) & 582 & I $3.3(4.0)$ & 365 & $<0.001$ & - \\
\hline Word recall $(C)$ & $5.8(2.1)$ & 594 & $5.0(2.3)$ & 395 & $<0.001$ & $0-16$ \\
\hline Mental rotations (D) & $5.5(1.7)$ & 557 & $5.4(1.6)$ & 343 & 0.420 & $0-10$ \\
\hline Digit span, forward (E) & $6.0(1.7)$ & 616 & $5.7(1.7)$ & 424 & 0.015 & $0-14$ \\
\hline Digit span, backward (E) & $4.9(1.8)$ & 613 & $4.5(1.9)$ & 421 & $<0.001$ & $0-14$ \\
\hline Synonym test $(\mathrm{F})$ & $19.1(6.2)$ & 605 & $17.2(6.3)$ & 385 & $<0.001$ & $0-30$ \\
\hline
\end{tabular}

Note: Differences in numbers could mainly be explained by vision impairment and dementia.

Abbreviations: SD, standard deviation; $n$, number; A, executive functioning; B, processing speed; C, episodic memory; D, spatial abilities; E, working memory; $\mathrm{F}$, semantic memory.

In the group that came to the reexamination session, the mean age at baseline was 83.0 years compared to 85.4 years in the drop-out group, $P<0.001$ (Table 1). The mean score on the LSI-A was higher for the reexamined group (a score of 25.9 compared to 22.8 for the drop-out group; $P<0.001)$. The reexamined group also had significantly higher mean values on all cognitive tests at baseline except for the mental rotation task when compared to the drop-out group (Table 1).

To explore potential confounding effects, correlations with the LSI-A at reexamination were calculated for the whole group as well as stratified for stroke "yes"/“no," and depressive mood high/low, education high/low, and independence/dependence in functional capacity using Pearson's correlation coefficient (Tables 2 and 3). For the whole group, digit cancellation, comparing figures, word recall, TMT B score, verbal fluency A, mental rotations, as well as verbal fluency animals and occupations were significantly correlated to LSI-A.

Correlations were also calculated for age groups 78-84 years and 87-93 years, dementia (yes/no), and sex (data not shown). While significant in the 78-84-year-old group, the verbal fluency tests and comparing figures lost association to LSI-A in the older group. In both sexes, there were similar significant associations between cognitive results and scores on the LSI-A. Stratification for dementia did not affect the correlations significantly.

Correlations were calculated between cognitive domains and LSI-A 3 years later (Table 5). Of the six cognitive domains, spatial abilities $\left(r=0.16, P<0.001 ; R^{2}=0.03\right)$ and processing speed $\left(r=0.15, P=0.001 ; R^{2}=0.02\right)$ showed the strongest correlations to LSI-A 3 years later. Executive abilities $(r=0.12)$ and episodic memory $(r=0.10)$ were also statistically significant at the $P<0.05$ level. Synonyms and working memory were not significantly related to LSI-A.

Multiple regression analyses were carried out for each cognitive domain separately with LSI-A at reexamination as the dependent variable. Each model was adjusted stepwise for sex, age, education, functional capacity, and depressive mood (Table 4). $R^{2}$ values for the final models were between 0.10 and 0.12 for the six different domains. Processing speed and spatial ability remained associated with LSI-A after adjustment, with $B=0.118, P=0.020$ for processing speed and $B=0.453, P=0.014$ for spatial ability.

\section{Discussion}

We found that Gf expressed by processing speed and spatial ability predicts LS 3 years later in the oldest-old. As mentioned in the introduction, most studies of associations between LS and cognition use rather coarse methods such as the MMSE that only detect a rather marked cognitive loss and typically only concern people with dementia. ${ }^{6}$ In this study, we showed that even when using cognitive instruments designed to measure aspects of intelligence in a healthy general population, there are clear associations with LS. We can thereby reject the null hypothesis even if the attributable fraction to LS is small. Spatial abilities and processing speed each explain less than 3\% each of variance in LS. From a public health perspective, factors not addressed in this study should thus be targeted firsthand when seeking to preserve 
Table 2 Pearson's correlation coefficients between cognitive variables at baseline and life satisfaction index A at reexamination with $P$-values in parentheses

\begin{tabular}{|c|c|c|c|c|c|}
\hline \multirow[t]{3}{*}{ Cognitive variable } & \multirow{3}{*}{$\frac{\text { All }}{r(P)}$} & \multirow{3}{*}{$\begin{array}{l}\text { Stroke } \\
r(P) \\
n=73\end{array}$} & \multirow{3}{*}{$\begin{array}{l}\text { No stroke } \\
r(P) \\
n=605\end{array}$} & \multirow{3}{*}{$\begin{array}{l}\text { No depressive mood } \\
r(P) \\
n=507\end{array}$} & \multirow{3}{*}{$\begin{array}{l}\text { Depressive mood } \\
r(P) \\
n=117\end{array}$} \\
\hline & & & & & \\
\hline & & & & & \\
\hline Trail Making Test $(\mathrm{A})$ & $-0.13(0.004)$ & $0.08(0.642)$ & $-0.14(0.003)$ & $-0.14(0.006)$ & $-0.08(0.46 \mathrm{I})$ \\
\hline Verbal fluency $F(A)$ & $0.07(0.133)$ & $0.06(0.679)$ & $0.06(0.223)$ & $0.04(0.393)$ & $0.25(0.014)$ \\
\hline Verbal fluency $A(A)$ & $0.10(0.033)$ & $0.01(0.967)$ & $0.09(0.050)$ & $0.06(0.217)$ & $0.28(0.006)$ \\
\hline Verbal fluency, animals (A) & $0.12(0.006)$ & $-0.10(0.503)$ & $0.13(0.005)$ & $0.07(0.130)$ & $0.29(0.005)$ \\
\hline Verbal fluency, occupations (A) & $0.13(0.003)$ & $-0.04(0.804)$ & $0.13(0.004)$ & $0.10(0.047)$ & $0.24(0.023)$ \\
\hline Confidence (A) & $0.07(0.086)$ & $-0.20(0.190)$ & $0.09(0.038)$ & $0.03(0.531)$ & $0.15(0.167)$ \\
\hline Digit cancellation (B) & $0.13(0.003)$ & $0.16(0.333)$ & $0.12(0.012)$ & $0.12(0.014)$ & $0.24(0.025)$ \\
\hline Comparing figures (B) & $0.17(<0.001)$ & $0.26(0.126)$ & $0.16(<0.00 \mathrm{I})$ & $0.18(<0.001)$ & $0.16(0.130)$ \\
\hline Word recall $(C)$ & $0.10(0.032)$ & $0.18(0.280)$ & $0.09(0.065)$ & $0.10(0.043)$ & $0.07(0.501)$ \\
\hline Mental rotations (D) & $0.16(<0.001)$ & $-0.16(0.353)$ & $0.18(<0.001)$ & $0.17(0.001)$ & $0.09(0.4 \mid 8)$ \\
\hline Digit span forward $(E)$ & $0.02(0.724)$ & $0.03(0.868)$ & $0.01(0.830)$ & $-0.02(0.667)$ & $0.18(0.091)$ \\
\hline Digit span backward (E) & $0.07(0.101)$ & $0.11(0.868)$ & $0.06(0.172)$ & $0.07(0.163)$ & $0.11(0.309)$ \\
\hline Synonym test $(F)$ & $0.03(0.045)$ & $-0.04(0.804)$ & $0.03(0.453)$ & $-0.01(0.813)$ & $0.15(0.142)$ \\
\hline
\end{tabular}

Note: Baseline variables for the whole study population as well as stratified for stroke and depressive mood.

Abbreviations: A, executive functioning; B, processing speed; C, episodic memory; D, spatial abilities; E, working memory; F, semantic memory.

Table 3 Pearson's correlation coefficients between the Life Satisfaction Index A at reexamination and cognitive variables at baseline stratified for education and functional capacity (ADL)

\begin{tabular}{|c|c|c|c|c|}
\hline \multirow[t]{3}{*}{ Cognitive variable } & \multirow{2}{*}{$\frac{\text { High education }}{r(P)}$} & \multirow{2}{*}{$\frac{\text { Low education }}{r(P)}$} & \multirow{2}{*}{$\begin{array}{l}\text { ADL, independent } \\
r(P)\end{array}$} & \multirow{3}{*}{$\begin{array}{l}\text { ADL, dependent } \\
r(P) \\
n=35 I\end{array}$} \\
\hline & & & & \\
\hline & $n=23 I$ & $n=436$ & $n=299$ & \\
\hline TMT B (A) & $-0.14(0.052)$ & $-0.13(0.021)$ & $-0.13(0.045)$ & $-0.10(0.14 I)$ \\
\hline Verbal fluency $F(A)$ & $0.05(0.475)$ & $0.02(0.666)$ & $-0.06(0.325)$ & $0.136(0.029)$ \\
\hline Verbal fluency $A(A)$ & $0.05(0.323)$ & $0.06(0.38 \mathrm{I})$ & $-0.02(0.738)$ & $0.15(0.013)$ \\
\hline Verbal fluency animals $(A)$ & $0.21(0.004)$ & $0.03(0.543)$ & $0.06(0.295)$ & $0.14(0.019)$ \\
\hline Verbal fluency occupations (A) & $0.15(0.037)$ & $0.07(0.196)$ & $-0.002(0.975)$ & $0.19(0.002)$ \\
\hline Confidence $(\mathrm{A})$ & $0.09(0.196)$ & $0.03(0.53 \mathrm{I})$ & $0.07(0.243)$ & $0.07(0.255)$ \\
\hline Comparing figures (B) & $0.13(0.084)$ & $0.17(0.003)$ & $0.11(0.08 I)$ & $0.21(0.001)$ \\
\hline Digit cancellation (B) & $0.11(0.15 I)$ & $0.14(0.015)$ & $0.04(0.524)$ & $0.16(0.013)$ \\
\hline Word recall $(\mathrm{C})$ & $0.14(0.062)$ & $0.05(0.355)$ & $0.12(0.052)$ & $0.02(0.724)$ \\
\hline Mental rotations (D) & $0.23(0.002)$ & $0.11(0.062)$ & $0.15(0.018)$ & $0.17(0.008)$ \\
\hline Digit span, forward $(E)$ & $-0.07(0.317)$ & $0.03(0.537)$ & $-0.06(0.301)$ & $0.04(0.500)$ \\
\hline Digit span, backward (E) & $0.07(0.309)$ & $0.03(0.552)$ & $-0.01(0.872)$ & $0.11(0.085)$ \\
\hline Synonyms (F) & $0.09(0.199)$ & $-0.04(0.452)$ & $-0.02(0.701)$ & $0.04(0.574)$ \\
\hline
\end{tabular}

Abbreviations: A, executive functioning; B, processing speed; C, episodic memory; D, spatial abilities; E, working memory; F, semantic memory.

Table 4 Linear regression models with the LSI-A at reexamination as the dependent variable and cognitive domains at baseline as the independent variables

\begin{tabular}{lllllll}
\hline LSI-A & Model I & Model 2 & Model 3 & Model 4 & Model 5 & Model 6 \\
\hline Executive abilities & $0.045(0.010)$ & $0.045(0.010)$ & $0.04 I(0.018)$ & $0.030(0.115)$ & $0.018(0.349)$ & $0.014(0.444)$ \\
Processing speed & $0.165(0.001)$ & $0.177(<0.001)$ & $0.161(0.001)$ & $0.139(0.006)$ & $0.117(0.020)$ & $0.118(0.020)$ \\
Episodic memory & $0.302(0.032)$ & $0.345(0.014)$ & $0.303(0.031)$ & $0.252(0.078)$ & $0.194(0.172)$ & $0.188(0.185)$ \\
Semantic memory & $0.038(0.445)$ & $0.044(0.368)$ & $0.033(0.502)$ & $-0.008(0.882)$ & $-0.033(0.518)$ & $-0.056(0.289)$ \\
Spatial abilities & $0.639(<0.00 I)$ & $0.569(0.002)$ & $0.554(0.002)$ & $0.498(0.007)$ & $0.505(0.006)$ & $0.453(0.014)$ \\
Working memory & $0.107(0.269)$ & $0.112(0.242)$ & $0.091(0.342)$ & $0.027(0.782)$ & $-0.018(0.856)$ & $-0.016(0.872)$ \\
\hline
\end{tabular}

Notes: The predictor variables were entered into the model separately in the following order: sex, age, education, functional capacity, and depressive mood. Represented are B-coefficients with $P$-values in parentheses. Depressive mood by CPRS was split into two groups, 0-6 and 7-34 points, independence/dependence in ADL, age in groups 78-84 years and 87-93 years. Model I is the cognitive domain. Model 2 is the cognitive domain + sex. Model 3 is the cognitive domain + sex + age. Model 4 is the cognitive domain + sex + age + education. Model 5 is the cognitive domain + sex + age + education + ADL. Model 6 is the cognitive domain + sex + age + education + ADL + CPRS. Abbreviations: LSI-A, Life Satisfaction Index A; CPRS, Comprehensive Psychiatric Rating Scale; ADL, activities of daily life. 
Table 5 Pearson's correlation coefficients between the six cognitive domains at baseline and LSI-A at reexamination

\begin{tabular}{lll}
\hline & LSI-A & $P$-value \\
\hline Executive abilities & 0.116 & 0.010 \\
Processing speed & 0.153 & 0.001 \\
Episodic memory & 0.095 & 0.032 \\
Semantic memory & 0.033 & 0.445 \\
Spatial abilities & 0.161 & $<0.001$ \\
Working memory & 0.048 & 0.269 \\
\hline
\end{tabular}

Abbreviation: LSI-A, Life Satisfaction Index A.

LS in the oldest-old. Examples of such factors could be burden of symptoms, which has been shown to explain 14\% of the variance in LS, ${ }^{1}$ functional capacity, and depressive mood. ${ }^{1,2}$ From a medical point of view, however, knowledge about the associations between cognition and LS might offer additional tools in an attempt to maintain LS in this patient group. The results of Wolinsky et al, ${ }^{9}$ who trained a group of elderly individuals in processing speed and saw a remarkable reduction in the risk of developing depressive symptoms, underline the clinical possibilities in this area.

Processing speed is "the ability to automatically and fluently perform relatively easy or over-learned elementary cognitive tasks, especially when high mental efficiency (eg, attention and focused concentration) is required. ${ }^{.25} \mathrm{In}$ our model (Table 4), one score higher on processing speed tests meant that LSI-A increased by 0.13 points. This domain is involved in processes such as driving and learning, as it involves perceiving stimuli and quickly being able to decide whether a given stimulus is important for the present situation or should be discarded. The connection to LS might be explained by the fact that impaired processing speed in the elderly could cause difficulties in the partaking of social and societal life.

Spatial ability has been defined as "the ability to generate, retain, retrieve and transform well-structured visual images." ${ }^{26}$ Because of their high demands on working memory, spatial tests are usually recognized as good measures of general intelligence. Spatial ability has been associated with creativity in various fields: artistic, mathematic, and scientific. A person with more spatial intelligence is assumed to create richer images of spoken or written language, and he or she is thereby better able to see contexts and more subtle aspects of topics. This may partly explain the association to LS found in this study. The regression model (Table 4) shows that scoring higher by one point on spatial abilities corresponds to an increase in LSI-A by about 0.5 points.

A lower cerebrovascular burden in the oldest-old is associated with longevity, fewer depressive symptoms, and stable cognitive function. ${ }^{27}$ Table 2 shows that the effect of certain cognitive tests (digit cancellation, comparing figures, mental rotations, and verbal fluency) remains in the healthy groups (ie, those not affected by hypertension, stroke, or dementia), meaning that the results are not based on a lower LS score due to effects of stroke or dementia per se.

In several of the cognitive tests, the correlations are higher for the group scoring higher on depressive mood (Table 2). This is probably an expression of the confounding effect of depressive mood on LS, even if there are still significant associations in the nondepressed group. Rabbitt et $\mathrm{l}^{28}$ found that higher scores on a depression scale (though the scores still fell in the "healthy" realm on the scale) yielded lower performance on measures of Gf as well as Gc. This could also be reciprocal with lower cognitive abilities, making an individual more prone to depressive mood and lower LS.

Confounding effects were illustrated by the higher correlations between cognition and LS in the functionally impaired group (Table 3). It may be the case that the functionally impaired group is more dependent on cognitive abilities when overcoming the threat to LS that impaired functionality constitutes. A previous study on stroke patients revealed that active coping strategies at a 3-year follow-up were related to greater improvement in both quality of life and ADL. ${ }^{29}$

Education is a possible confounder since it has been shown to be associated to LS and cognition. ${ }^{1,30}$ Cognitive abilities seem to affect LS more in those with higher levels of education (Table 3 ). This might reflect that a person with higher intelligence has built his/her life around relations and activities that demand higher intellectual capacities. However, after adjusting for education in the regression models, Gf still affected LS. Our results thus support those of Moore et $\mathrm{al}^{30}$ who found positive correlations between cognitive performance and higher education, as well as between higher income and self-rated successful aging. For verbal fluency, the associations are much weaker for the group with lower education levels than for those with higher education levels. The verbal fluency tests are affected by education, and when the model is adjusted for education, the association between verbal fluency and LS is lost.

Impaired LS and cognitive decline have been shown to be a part of the concept of "terminal decline." " 11,32 A potential weakness in this study is that age was measured as time from birth; adjusting for proximity to death might have diminished possibilities for confounding, making the association between Gf and LS weaker. 
Contrary to results of Siedlecki et al, ${ }^{10}$ we found associations between cognitive domains related to Gf and LS. Even if the elderly individual is retired in most cases and thereby does not face the ever-changing situations associated with work and career, this might reflect that the individual still has to be able to learn new things constantly to keep up with changes in society and to face new living conditions and perhaps diminishing physical capabilities that the aging body presents. The only test in our battery indicating Gc, the synonym-test, was not significantly associated with LS, just as in the results of Siedlecki et al. ${ }^{10}$

Many different instruments and different variants of these instruments are in use in this research area; therefore, data for specific cognitive tests were presented in this paper so as to make comparisons with other studies easier. To reduce the risk of statistical mass significance, the tests were categorized into cognitive domains in the final analysis.

The study population was large and randomly selected using the Swedish National Population Registry. No exclusion was made based on living conditions; home visits were made in almost every third case to reduce selection bias. Help with interpretation and filling in questionnaires was offered to people with language difficulties and other disabilities.

Attrition analysis showed that younger participants with higher scores on the LSI-A index and higher scores on the cognitive tests were those that continued on in the study; therefore, the generalizability of the results should be made with caution. However, a rather large attrition rate is common in studies that include the oldest-old. ${ }^{13}$ Given that the majority of individuals lost to follow-up were those with lower LS and who presented with lower scores on the cognitive tests, it is likely that the attrition reduced the overall distribution, and as a consequence, the correlations noted in this study might be an underestimation of the true association.

Different definitions are used when it comes to the oldestold, with some definitions drawing the line at age 80 years and some at 85 years. In the Good Aging in Skåne study, individuals aged 78 years and older are reexamined every 3 years, which is in contrast to younger participants who are reexamined every 6 years. To gain power in the study, we included those participants that would pass the age of 80 years during the 3 years between examinations.

In conclusion, fluid cognitive abilities (especially spatial abilities and perceptual speed) predict LS in the oldest-old. Several studies have shown that through training, fluid cognitive abilities can be enhanced to a significant and meaningful degree even in very old ages..$^{9,28}$ These findings perhaps implicate additional roads for professionals working with the oldest-old and seeking to preserve these individuals' LS.

\section{Acknowledgments}

The project "Good Aging in Skåne" (www.med.lu.se/hvs/ geriatrik), part of the Swedish National study on Aging and Care (www.snac.org), was supported by the Swedish Ministry of Health and Social Affairs, the county Region Skåne, the Medical Faculty at Lund University, and the Vårdal Institute.

\section{Author Contributions}

All three authors contributed substantially to the design of the study as well as to analysis and interpretation of data. $\AA$. Enkvist wrote the article, but all three authors took part in the writing process through critical revision and final approval of the manuscript.

\section{Disclosure}

The authors report no conflicts of interest in this work.

\section{References}

1. Enkvist A, Ekström H, Elmståhl S. What factors affect life satisfaction (LS) among the oldest-old? Arch Gerontol Geriatr. 2012;54(1): 140-145.

2. Enkvist Å, Ekström H, Elmståhl S. Life satisfaction (LS) and symptoms among the oldest-old: results from the longitudinal population study called Good Aging in Skåne. Arch Gerontol Geriatr. 2012;54(1): $146-150$.

3. Berg AI, Hoffman L, Hassing LB, McClearn GE, Johansson B. What matters, and what matters most, for change in life satisfaction in the oldest-old? A study over 6 years among individuals 80+. Aging Ment Health. 2009;13(2):191-201.

4. Gerstorf D, Ram N, Röcke C, Lindenberger U, Smith J. Decline in life satisfaction in old age: longitudinal evidence for links to distance-fromdeath. Psychol Aging. 2008;23(1):154-168.

5. Jones T, Rapport L, Hanks R, Lichtenberg P, Telmet K. Cognitive and psychosocial predictors of subjective well-being in urban older adults. Clin Neuropsychol. 2003;17(1):3-18.

6. St John PD, Montgomery PR. Cognitive impairment and life satisfaction in older adults. Int J Geriatr Psychiatry. 2010;25(8):814-821.

7. Baltes PB. The aging mind: potentials and limits. Gerontologist. 1993;33(5):580-594.

8. Cattell RB. Abilities: Their Structure, Growth, and Action. New York, NY: Houghton Mifflin; 1971.

9. Wolinsky FD, Vander Weg MW, Martin R, et al. The effect of speed-ofprocessing training on depressive symptoms in ACTIVE. J Gerontol A Biol Sci Med Sci. 2009;64(4):468-472.

10. Siedlecki KL, Tucker-Drob EM, Oishi S, Salthouse TA. Life satisfaction across adulthood: different determinants at different ages? J Posit Psychol. 2008;3(3):153-164.

11. Baltes PB, Baltes MM. Successful Aging: Perspectives from the Behavioral Sciences. Cambridge, UK: Cambridge University Press; 1990.

12. Ekström H, Elmståhl S. Pain and fractures are independently related to lower walking speed and grip strength: results from the population study "Good Ageing in Skåne". Acta Orthop. 2006;77(6):902-911. 
13. Borglin G, Jakobsson U, Edberg AK, Hallberg IR. Self-reported health complaints and their prediction of overall and health-related quality of life among elderly people. Int J Nurs Stud. 2004;42(2):147-158.

14. Neugarten BL, Havighurst RJ, Tobin SS. The measurement of life satisfaction. J Gerontol. 1961;16:134-143.

15. Lobello SG, Underhill AT, Fine PR. The reliability and validity of the Life Satisfaction Index-A with survivors of traumatic brain injury. Brain Inj. 2004;18(11):1127-1134.

16. Lezak MD, Howieson DB, Loring DW. Neuropsychological Assessment. 4th ed. New York, NY: Oxford University Press; 2004.

17. Dureman I, Sälde H. [Psychometric methods for clinical use]. DSBatteriet. Manual. SRB:1. Stockholm, Sweden: Psykologiförlaget; 1960. Swedish.

18. Lewis RF, Rennick PM. Manual for the Repeatable CognitivePerceptual-Motor Battery. Grosse Pointe Park, MI: Axon; 1979.

19. Allwood CM. Confidence in own and others' knowledge. Scand J Psychol. 1994;35(3):198-211.

20. Wechsler D. Weschsler Adult Intelligence Scale-III. San Antonio, TX: The Psychological Corporation; 1997.

21. Shepard RN, Metzler J. Mental rotations of three-dimensional objects. Science. 1971;171(3972):701-703.

22. Montgomery SA, Asberg M. A new depression scale designed to be sensitive to change. Br J Psychiatry. 1979;134:382-389.

23. Snaith RP, Harrop FM, Newby DA, Teale C. Grade scores of the Montgomery-Asberg Depression and the Clinical Anxiety Scales. Br J Psychiatry. 1986;148:599-601.

24. Sonn U, Asberg KH. Assessment of activities of daily living in the elderly. A study of 76-year-olds in Gothenburg, Sweden. Scand J Rehabil Med. 1991;23(4):193-202.
25. McGrew KS. CHC theory and the human cognitive abilities project: standing on the shoulders of the giants of psychometric intelligence research. Intelligence. 2009;37(1):1-10.

26. Lohman DF. Spatial ability and G. In: Dennis I, Tapsfield P, editors. Human Abilities: Their Nature and Assessment. Hillsdale, NJ: Erlbaum; 1996:97-116.

27. Paulson D, Bowen ME, Lichtenberg PA. Successful aging and longevity in older old women: the role of depression and cognition. JAging Res. 2011;2011:912680.

28. Rabbitt P, Lunn M, Ibrahim S, Cobain M, McInnes L. Unhappiness, health and cognitive ability in old age. Psychol Med. 2008;38(2):229-236.

29. Elmståhl S, Sommer M, Hagberg B. A 3-year follow-up of stroke patients: relationships between activities of daily living and personality characteristics. Arch Gerontol Geriatr. 1996;22(3):233-244.

30. Moore DJ, Sitzer D, Depp CA, et al. Self-administered cognitive screening for a study of successful aging among community-dwelling seniors: a preliminary study. Int J Geriatr Psychiatry. 2007;22(4):327-331.

31. Mroczek DK, Spiro 3rd A. Change in life satisfaction during adulthood: Findings from the veterans' affairs normative aging study. Journal of Personality and Social Psychology. 2005;88(1):189-202.

32. Kleemeier RW. Intellectual changes in the senium. Proceedings of the American Statistical Association. 1962;1:181-190.
Clinical Interventions in Aging

\section{Publish your work in this journal}

Clinical Interventions in Aging is an international, peer-reviewed journal focusing on evidence-based reports on the value or lack thereof of treatments intended to prevent or delay the onset of maladaptive correlates of aging in human beings. This journal is indexed on PubMed Central, MedLine, the American Chemical Society's 'Chemical Abstracts

\section{Dovepress}

Service' (CAS), Scopus and the Elsevier Bibliographic databases. The manuscript management system is completely online and includes a very quick and fair peer-review system, which is all easy to use. Visit http://www.dovepress.com/testimonials.php to read real quotes from published authors. 